A. ROS

KODAI MATH. J.

6 (1983), $88-99$

\title{
SPECTRAL GEOMETRY OF CR-MINIMAL SUBMANIFOLDS IN THE COMPLEX PROJECTIVE SPACE
}

\author{
By Antonio Ros
}

Introduction. In the first part of this paper we will study an isometric imbedding of the complex projective space in the Euclidean space, see [7].

In the second part we use this imbedding and the total mean curvature theory, see [4], in order to obtain certain boundaries of the volume and the first eigenvalue of the spectrum of $C R$-minimal closed submanifolds of the complex projective space, such as certain characterizations of some of these submanifolds, in function of these geometric invariants. We give a $\lambda_{1}$-characterization of totally geodesic complex submanifolds, a spectral reduction of codimension theorem for totally real submanifolds and some other results.

Manifolds are assumed to be connected and dimension $n \geqq 2$ unless mentioned otherwise. For the necessary knowledge and notations of the geometry of submanifolds, see [2], and for spectral geometry, see [1].

1. An imbedding of the complex projective space in the Euclidean space.

Let $H M(n)=\left\{A \in g l(n, C) / \bar{A}=A^{t}\right\}$ be the set of $n \times n$-Hermitian matrices. $H M(n)$ is a $n^{2}$-dimensional linear subspace of $g l(n, C)$. We define in $H M(n)$ the metric

$$
g(A, B)=2 \operatorname{trace}(A B) \text { for all } A, B \text { in } H M(n) .
$$

Let $C P^{n}=\{A \in H M(n+1) / A A=A$, trace $A=1\}$ and $U(n)$ be the unitary group.

LEMMA 1.1. $C P^{n}$ is a submanifold of $H M(n+1)$ diffeomorphic to $U(n+1) / U(1) \times U(n)$.

Proof. Let $A$ be in $C P^{n}$. Since $A$ is a Hermitian matrix, there exists $P$ in $U(n+1)$ such that

$$
P A P^{-1}=\left(\begin{array}{ccc}
h_{0} & & \\
& \ddots & \\
& & h_{n}
\end{array}\right) .
$$

As $P A P^{-1}=\left(P A P^{-1}\right)^{2}, h_{\imath}=h_{\imath}^{2}$, so that $h_{\imath}=0$ or $h_{\imath}=1$, but $\operatorname{trace}\left(P A P^{-1}\right)=1$, therefore there exists an index $\imath_{0}$ such that $h_{\imath_{0}}=1$ and $h_{\imath}=0$ for all $\imath \neq \imath_{0}$.

Received March 25, 1982 
Hence, we see that there exists $P$ in $U(n+1)$ such that

$$
P A P^{-1}=\left(\begin{array}{cccc}
1 & & & \\
& 0 & & \\
& & \ddots & \\
& & & 0
\end{array}\right)=A_{0} .
$$

We will say that $A_{0}$ is the origin of $C P^{n}$. Moreover $C P^{n}$ is the orbit of $A_{0}$ for the action of $U(n+1)$ over $H M(n+1)$ given by $(P, A) \mapsto P A P^{-1}$, where $P$ is in $U(n+1)$ and $A$ is in $H M(n+1)$. The isotropy subgroup of $A_{0}$ is $U(1) \times U(n)$. Therefore $C P^{n} \cong U(n+1) / U(1) \times U(n)$.

(Q.E.D.)

For any $A$ in $C P^{n}$, we denote by $T_{A}\left(C P^{n}\right)$ the tanget space of $C P^{n}$ at $A$ identified by means of the immersion with a subspace of $H M(n+1)$. In the same way we denote by $T_{A}^{\perp}\left(\boldsymbol{C} P^{n}\right)$ the normal space of $\boldsymbol{C} P^{n}$ in $H M(n+1)$ at the point $A$.

LeMma 1.2. For any point $A$ in $C P^{n}$, we have

$$
\begin{gathered}
T_{A}\left(\boldsymbol{C} P^{n}\right)=\{X \in H M(n+1) / X A+A X=X\}, \\
T_{A}^{\perp}\left(\boldsymbol{C} P^{n}\right)=\{Z \in H M(n+1) / A Z=Z A\} .
\end{gathered}
$$

Proof. Let $\alpha: \Gamma \rightarrow C P^{n}$ be a curve such that $\alpha(0)=A$ and $\alpha^{\prime}(0)=X$, where $\Gamma$ will denote an open interval of real numbers which contains 0 . Then from $\alpha(t) \alpha(t)=\alpha(t)$ we obtain $X A+A X=X$. Therefore we have one inclusion. Since the applications $L_{p}: H M(n+1) \rightarrow H M(n+1)$ given by $A \mapsto P A P^{-1}$, where $P$ is in $U(n+1)$, are isometries, it is enough to establish this equalities at the origin. Now we will compute the dimension of the subspace $\left\{X \in H M(n+1) / X A_{0}+\right.$ $\left.A_{0} X=X\right\}$.

For any $X \in H M(n+1)$ we put

$$
X=\left(\begin{array}{cc}
a & b \\
\bar{b}^{t} & c
\end{array}\right) \quad \text { where } \quad a \in \boldsymbol{R}, b \in \boldsymbol{C}^{n} \text { and } c \in H M(n) .
$$

Then $X A_{0}+A_{0} X=X$ if and only if $a=0$ and $c=0$, so that

$$
X=\left(\begin{array}{cc}
0 & b \\
\bar{b}^{t} & 0
\end{array}\right), \quad \text { with } \quad b \in C^{n} .
$$

The real dimension of this subspace is $2 n=\operatorname{dim} T_{A}\left(\boldsymbol{C} P^{n}\right)=\operatorname{dim} U(n+1) / U(1)$ $\times U(n)$ and so we have (1.1). Let

A vector $Z$ is in $T_{A_{0}}^{\perp}\left(C P^{n}\right)$ if and only if $2 \operatorname{trace}(X Z)=0$ for all $X \in T_{A_{0}}\left(\boldsymbol{C} P^{n}\right)$.

$$
Z=\left(\begin{array}{cc}
x & y \\
\bar{y}^{t} & z
\end{array}\right)
$$


Then, 2 trace $(X Z)=4$ Real trace $\left(b \bar{y}^{t}\right)$. Therefore $g(X, Z)=0$ for all $X$ in $T_{A_{0}}\left(C P^{n}\right)$, if and only if $y=0$.

On the other hand, $Z A_{0}=A_{0} Z$ if and only if $y=0$.

Remark 1.3. The vector fields given by $A \mapsto A$ and $A \mapsto I$ (where $I$ denotes the identity matrix) are normal to $C P^{n}$. The vector fields given by $A \mapsto A Q$ $+Q A-2 A Q A$ are tangent to $C P^{n}$ for all $Q$ in $H M(n+1)$.

Hence forth, we will use the following relations which can be obtained by direct calculus. Let $A$ be in $\boldsymbol{C} P^{n}$ and $X, Y$ in $T_{A}\left(\boldsymbol{C} P^{n}\right)$. Then $A X Y=X Y A$, $A X A=0, X(I-2 A)=-(I-2 A) X,(I-2 A)^{2}=I,(I-2 A) X Y=X Y(I-2 A)$.

Proposition 1.4. Let $D$ be the Riemannian connection of $H M(n+1), \nabla$ the induced connection in $\mathrm{CP}^{n}$, $\tilde{\sigma}$ the second fundamental form of the immersion, $\nabla^{\perp}$ and $\boldsymbol{A}$ the normal connection and the Werngarten endomorphism and $\tilde{H}$ the mean curvature vector of $\boldsymbol{C} P^{n}$. Then

$$
\begin{gathered}
\nabla_{X} Y=A\left(D_{X} Y\right)+\left(D_{X} Y\right) A-2 A\left(D_{X} Y\right) A, \\
\tilde{\sigma}(X, Y)=(X Y+Y X)(I-2 A), \\
\nabla_{X}^{\perp} Z=D_{X} Z+2 A\left(D_{X} Z\right) A-\left(D_{X} Z\right) A-A\left(D_{X} Z\right), \\
A_{Z} X=(X Z-Z X)(I-2 A), \\
\tilde{H}=\frac{1}{2 n}[I-(n+1) A],
\end{gathered}
$$

where $X$ and $Y$ are tangent vector fields to $C P^{n}$, and $Z$ is a normal vector field to $C P^{n}$.

Proof. Let $\nabla$ and $\tilde{\sigma}$ be as in (1.3) and (1.4). Let $X$ be any vector in $T_{A}\left(\boldsymbol{C} P^{n}\right)$ and $Y$ any tangent vector field to $\boldsymbol{C} P^{n}$. If $\alpha: \Gamma \rightarrow \boldsymbol{C} P^{n}$ is a curve which satisfies $\alpha(0)=A$ and $\alpha^{\prime}(0)=X$, we have $\alpha(t) Y(t)+Y(t) \alpha(t)=Y(t)$. Therefore

$$
X Y+Y X+A\left(D_{X} Y\right)+\left(D_{X} Y\right) A=D_{X} Y .
$$

On the other hand, we have $\alpha(t) Y(t) \alpha(t)=0$. Therefore

$$
X Y A+A\left(D_{X} Y\right) A+A Y X=0 .
$$

From (1.8) and (1.9), we get $D_{X} Y=\nabla_{X} Y+\tilde{\sigma}(X, Y)$.

A simple calculations proves that $\nabla_{X} Y$ (resp. $\tilde{\sigma}(X, Y)$ ) is tangent (resp. normal) to $\boldsymbol{C} P^{n}$. Then we have (1.3) and (1.4).

Let $\nabla^{\perp}$ and $\boldsymbol{A}$ be as in (1.5) and (1.6). Let $Z$ be any normal vector field to $C P^{n}$. We have $\alpha(t) Z(t)=Z(t) \alpha(t)$, then

$$
\begin{gathered}
X Z+A\left(D_{X} Z\right)-\left(D_{X} Z\right) A-Z X=0, \\
A_{Z} X=(X Z-Z X)(I-2 A)=\left[\left(D_{X} Z\right) A-A\left(D_{X} Z\right)\right](I-2 A) \\
=2 A\left(D_{X} Z\right) A-\left(D_{X} Z\right) A-A\left(D_{X} Z\right)=\nabla_{X}^{\perp} Z-D_{X} Z .
\end{gathered}
$$


From (1.1) (resp. (1.2)) we see that $\boldsymbol{A}_{Z} X$ (resp. $\nabla_{X}^{\perp} Z$ ) is tangent (resp. normal), hence we have (1.5) and (1.6).

It is enough, to verify (1.7) at the origin.

Let $\left\{E_{1}, \cdots E_{n}, E_{1}^{*}, \cdots E_{n}^{*}\right\}$ be an orthonormal base in $T_{A}\left(\boldsymbol{C} P^{n}\right)$ defined by

$$
\begin{aligned}
& E_{k}=\frac{1}{2}(k)\left(\begin{array}{cccccccc}
0 & 0 & \cdots & 0 & 1 & 0 & \cdots & 0 \\
\hdashline 0 & & & & & & & \\
\vdots & & & & & & & \\
1 & & & & 0 & & & \\
\vdots & & & & & & & \\
0 & & & & & & &
\end{array}\right), \\
& \text { (k) } \\
& E_{k}^{*}=\frac{\sqrt{-1}}{2}(k)\left(\begin{array}{c:cccccc}
0 & 0 & \cdots & 0 & 1 & \cdots & 0 \\
\hdashline 0 & & & & & & \\
\vdots & & & & & & \\
-1 & & & & 0 & & \\
\vdots & & & & & & \\
0 & & & & & &
\end{array}\right) \text {. }
\end{aligned}
$$

A direct calculation proves that

$$
\widetilde{H}_{A_{0}}=\frac{1}{2 n}\left(\begin{array}{c|ccc}
-n & 0 & \cdots & 0 \\
\hline 0 & 1 & & \\
\vdots & & \ddots & \\
0 & & & 1
\end{array}\right)=\frac{1}{2 n}\left[I-(n+1) A_{0}\right] \text {. (Q. E. D.) }
$$

LEMMA 1.5. a) Let $f$ be the diffeomorphism obtained in lemma 1.1. Then $f$ is an isometry when we consider on $U(n+1) / U(1) \times U(n)$ the Fubini-Study metruc with holomorphic sectional curvature $c=1$, and on $C P^{n}$ the metric induced by that on $H M(n+1)$.

b) The complex structure induced by the isometry $f$ in $\boldsymbol{C} P^{n}$ is given by $J X=\sqrt{-1}(I-2 A) X$, for all $X$ in $T_{A}\left(C P^{n}\right)$.

Proof. a) Since both metrics are $U(n+1)$-invariant, it is enough to see that the differential of $f$ at the origin is an isometry between the corresponding tangent spaces.

Let $[P]$ be the coset of $P \in U(n+1)$ in $U(n+1) / U(1) \times U(n)$. Then $f([P])$ $=P A_{0} P^{-1}$ and so

$$
T_{0}(U(n+1) / U(1) \times U(n))=\left\{\left(\begin{array}{rr}
0 & a \\
-\bar{a}^{t} & 0
\end{array}\right) / a \in \boldsymbol{C}^{n}\right\}, \quad 0=[I] .
$$


The Fubini-Study metric of the constant holomorphic sectional curvature $c=1$ at the origin is given by

$$
g_{0}\left(\left(\begin{array}{rr}
0 & a \\
-\bar{a}^{t} & 0
\end{array}\right),\left(\begin{array}{rr}
0 & b \\
-\bar{b}^{t} & 0
\end{array}\right)\right)=2 \operatorname{trace}\left(\begin{array}{cc}
a \bar{b}^{t} & 0 \\
0 & \bar{a}^{t} b
\end{array}\right) .
$$

Let $\alpha: \Gamma \rightarrow U(n+1)$ be a curve such that $\alpha(0)=I$ and $\alpha^{\prime}(0)=\left(\begin{array}{cc}0 & a \\ -\bar{a}^{t} & 0\end{array}\right)$. We consider the curve $\beta: \Gamma \rightarrow U(n+1) / U(1) \times U(n)$ given by $\beta(t)=[\alpha(t)]$. $d f_{0}\left(\begin{array}{cc}0 & a \\ -\bar{a}^{t} & 0\end{array}\right)=(f \beta)^{\prime}(0)=\alpha^{\prime}(0) A_{0} \overline{\alpha(0)^{t}}+\alpha(0) A_{0} \overline{\alpha^{\prime}(0)^{t}}=\left(\begin{array}{rr}0 & -a \\ -\bar{a}^{t} & 0\end{array}\right)$, and we have $g_{A_{0}}\left((d f)_{0}\left(\begin{array}{cc}0 & a \\ -\bar{a}^{t} & 0\end{array}\right),(d f)_{0}\left(\begin{array}{rr}0 & b \\ -\bar{b}^{t} & 0\end{array}\right)\right)=2 \operatorname{trace}\left(\begin{array}{cc}a \bar{b}^{t} & 0 \\ 0 & \bar{a}^{t} b\end{array}\right)$. This show a).

b) The complex structure $\tilde{J}$ at the origin of $U(n+1) / U(1) \times U(n)$ is given by $\tilde{J}\left(\begin{array}{cc}0 & a \\ -\bar{a}^{t} & 0\end{array}\right)=\sqrt{ }-1\left(\begin{array}{rr}0 & -a \\ -\bar{a}^{t} & 0\end{array}\right)$, see [6]. Let $\left(\begin{array}{cc}0 & a \\ \bar{a}^{t} & 0\end{array}\right)$ be a vector in $T_{A_{0}}\left(\boldsymbol{C} P^{n}\right)$. Therefore the complex structure induced in $\boldsymbol{C} P^{n}$ is given by

$$
J\left(\begin{array}{cc}
0 & a \\
\bar{a}^{t} & 0
\end{array}\right)=d f_{0} \tilde{J}\left(d f_{0}\right)^{-1}\left(\begin{array}{cc}
0 & a \\
\bar{a}^{t} & 0
\end{array}\right)=\sqrt{ }-1\left(\begin{array}{rr}
0 & -a \\
\bar{a}^{t} & 0
\end{array}\right) .
$$

On the other hand

$$
\left.\sqrt{-1}\left(I-2 A_{0}\right)\left(\begin{array}{cc}
0 & a \\
\bar{a}^{t} & 0
\end{array}\right)=\sqrt{-1}\left(\begin{array}{rr}
0 & -a \\
\bar{a}^{t} & 0
\end{array}\right) . \quad \text { (Q.E. D. }\right)
$$

The following proposition resumes some properties of the immersion. For other properties, see [5], [7].

Proposition 1.6. The immersion of $\boldsymbol{C P}^{n}$ in $H M(n+1)$ verifies the following properties.

a) It is an isometric $U(n+1)$-equivariant imbedding.

b) $\tilde{\sigma}(J X, J Y)=\tilde{\sigma}(X, Y)$ and $\nabla \tilde{\sigma}=0$, that is, the second fundamental form is parallel.

c) It is minimal in the sphere $S$, whose center is $[1 /(n+1)] I$ and whose radius is $\sqrt{ } 2 n /(n+1)$.

Proof. a) It is a consequence of lemma 1.1 and 1.5 .

b) It is easy to see that $\tilde{\sigma}(J X, J Y)=\tilde{\sigma}(X, Y)$ for all $X, Y \in T_{A}\left(C P^{n}\right)$. Let $X, Y_{1}, Y_{2}$ be any three vector fields tangent to $C P^{n}$. Then we have

$$
\begin{aligned}
(\nabla \tilde{\sigma})_{X}\left(J Y_{1}, J Y_{2}\right) & =\nabla_{X} \tilde{\sigma}\left(J Y_{1}, J Y_{2}\right)-\tilde{\sigma}\left(\nabla_{X} J Y_{1}, J Y_{2}\right)-\tilde{\sigma}\left(J Y_{1}, \nabla_{X} J Y_{2}\right) \\
& =\nabla_{X} \tilde{\sigma}\left(Y_{1}, Y_{2}\right)-\tilde{\sigma}\left(\nabla_{X} Y_{1}, Y_{2}\right)-\tilde{\sigma}\left(Y_{1}, \nabla_{X} Y_{2}\right)
\end{aligned}
$$




$$
=(\nabla \tilde{\sigma})_{X}\left(Y_{1}, Y_{2}\right) \text {. }
$$

Therefore we have $(\nabla \tilde{\sigma})_{X}(Y, J Y)=0$, for all $Y$ in $T_{A}\left(\boldsymbol{C} P^{n}\right)$, and so from Codazzi's equation $(\nabla \tilde{\sigma})_{Y}(X, J Y)=0$. If we choose $X=J Y$, we have $0=(\nabla \tilde{\sigma})_{Y}(J Y, J Y)=(\nabla \tilde{\sigma})_{Y}(Y, Y)$. Hence $\nabla \tilde{\sigma}=0$.

c) If $A$ is in $C P^{n}$ then $g\left(A-\frac{1}{n+1} I, A-\frac{1}{n+1} I\right)=\frac{2 n}{n+1}$. Therefore $\boldsymbol{C} P^{n}$ is included in $S$. Let $\widetilde{H}$ be the mean curvature vector of $\boldsymbol{C} P^{n}$ in $H M(n+1)$. $\widetilde{H}=\frac{1}{2 n}[I-(n+1) A]=-\frac{n+1}{2 n}\left(A-\frac{1}{n+1} I\right)$. Therefore $C P^{n}$ is minimal in $S$, see $[2]$.

LEMma 1.7. Let $E_{1}, E_{2}$ be any two vectors in $T_{A}\left(C P^{n}\right)$ such that $g\left(E_{1}, E_{2}\right)=0$ and $g\left(E_{1}, E_{1}\right)=g\left(E_{2}, E_{2}\right)=1$. Then
a) $g\left(\tilde{\sigma}\left(E_{1}, E_{1}\right), \tilde{\sigma}\left(E_{1}, E_{1}\right)\right)=1$,
b) $1 / 2 \leqq g\left(\tilde{\sigma}\left(E_{1}, E_{1}\right), \tilde{\sigma}\left(E_{2}, E_{2}\right)\right) \leqq 1$.

Moreover if we have $g\left(E_{1}, J E_{2}\right)=0$, then

c) $g\left(\tilde{\sigma}\left(E_{1}, E_{1}\right), \tilde{\sigma}\left(E_{2}, E_{2}\right)\right)=1 / 2$,

d) $g\left(\tilde{\sigma}\left(E_{1}, E_{2}\right), \tilde{\sigma}\left(E_{1}, E_{2}\right)\right)=1 / 4$.

Proof. Let $E_{1}=\left(\begin{array}{cc}0 & a \\ \bar{a}^{t} & 0\end{array}\right)$ and $E_{2}=\left(\begin{array}{cc}0 & b \\ \bar{b}^{t} & 0\end{array}\right)$. Then $g\left(E_{1}, E_{1}\right)=1$ if and only if $a \bar{a}^{t}=1 / 4, g\left(E_{1}, E_{2}\right)=0$ if and only if $a \bar{b}^{t}=\sqrt{-1} h$, where $h \in \boldsymbol{R}$. Moreover $g\left(E_{1}, J E_{2}\right)=0$ if and only if $a \bar{b}^{t}=0$. Now a), c) and d) are obvious.

b) $g\left(\tilde{\sigma}\left(E_{1}, E_{1}\right), \tilde{\sigma}\left(E_{2}, E_{2}\right)\right)=8 \operatorname{trace}\left(E_{1}^{2} E_{2}^{2}\right)=8 \operatorname{trace}\left(\begin{array}{cc}1 / 16 & 0 \\ 0 & \sqrt{-1} h \bar{a}^{t} b\end{array}\right)=1 / 2$ $+8 h^{2}$. But $h^{2}=\left|a \bar{b}^{t}\right|^{2} \leqq|a|^{2}|b|^{2}=1 / 16$.

(Q.E.D.)

\section{2. $C R$-minimal submanifolds in the complex projective space.}

For $C R$-submanifolds see for example [4]. In the following we write $M^{2 n+p}$ for a $C R$-submanifold of $C P^{n}$, where $2 n=\operatorname{dim} \mathscr{D}$ and $p=\operatorname{dim} \mathscr{D}^{\perp}, \mathscr{D}$ being the holomorphic distribution and $\mathscr{D}^{\perp}$ the totally real distribution of $M$.

LEMMA 2.1. a) Let $M^{n}$ be a submanifold of $C P^{m}$. Let $H^{\perp}$ be the normal component of the mean curvature vector of $M^{n}$ in $H M(m+1)$ to $C P^{m}$. Then

$$
(n+1) / 2 n \leqq g\left(H^{\perp}, H^{\perp}\right) \leqq 1 .
$$

b) Let $M^{2 n+p}$ be a CR-submanfold of $C P^{m}$. Let $H^{\perp}$ be as in a). Then 


$$
g\left(H^{\perp}, H^{\perp}\right)=\left[(2 n+p)^{2}+4 n+p\right] / 2(2 n+p)^{2} .
$$

Proof. a) Let $\left\{E_{1}, \cdots E_{n}\right\}$ be an orthonormal base of $T_{A}\left(M^{n}\right)$ where $A$ is any point in $M^{n}$. Let $\tilde{\sigma}$ be the second fundamental form of $C P^{m}$ in $H M(m+1)$. Then $H^{\perp}=\frac{1}{n} \sum_{\imath} \tilde{\sigma}\left(E_{\imath}, E_{\imath}\right)$. By using lemma 1.7 we have (2.1).

b) We can choose an orthonormal base of $T_{A}(M)$ of the type $\left\{E_{1}, \cdots E_{n}\right.$, $\left.J E_{1}, \cdots J E_{n}, F_{1}, \cdots F_{p}\right\}$, where $E_{\imath}, J E_{\imath}$ are in $\mathscr{D}$ and $F_{\jmath}$ is in $\mathscr{D}^{\perp}$. From lemma 1.7 , we have $(2.2)$.

(Q.E. D.)

LEMMA 2.2. Let $M^{2 n+p}$ be a CR-submanifold of $C P^{m}$, $\tilde{\sigma}$ the second fundamental form of $\boldsymbol{C P} P^{m}$ in $H M(m+1)$ and $\tilde{\sigma}_{M}$ its restriction to $M$. Then

$$
g\left(\tilde{\sigma}_{M}, \tilde{\sigma}_{M}\right)=(1 / 4)\left[(2 n+p)^{2}+4 n+3 p\right] .
$$

The proof can be obtained by using lemma 1.7. From the expresion of the scalar curvature for submanifolds in the Euclidean space, we obtain the following

Corollary 2.3. Let $M^{2 n+p}$ be a CR-submanifold of $\boldsymbol{C P} P^{m}$. Let $H$ be the mean curvature vector of $M^{2 n+p}$ in $C P^{m}, r$ the scalar curvature of $M^{2 n+p}$, and $\sigma$ the second fundamental form of $M^{2 n+p}$ in $C P^{m}$. Then

$$
r=\left[(2 n+p)^{2}+4 n-p\right] / 4+(2 n+p)^{2} g(H, H)-g(\sigma, \sigma) .
$$

B. Y. Chen has proved the following theorems:

Theorem A. [2]. Let $M$ be an n-dimensional closed submanifold of $E^{m}$. Then we have

$$
\int_{M} \alpha^{n} d v \geqq c_{n}
$$

where $\alpha=\sqrt{ } g(\bar{H}, H)$ is the mean curvature of $M$ and $c_{n}$ is the volume of unt $n$-sphere. The equality holds if and only if $M$ is imbedded as an ordinary $n$ sphere in an affine $(n+1)$-space.

For an isometric immersion of a closed manifold $M$ in the Euclidean space $x: M \rightarrow E^{m}$, we put $x=\left(x_{1}, \cdots x_{m}\right)$, where $x_{\imath}$ is the $i$-th coordinate function of $M$ in $E^{m}$. We call an isometric immersion $x$ is of order $k$ if each coordinate function $x_{\imath}$ of $x$ is an eigenfunction of the Laplace Beltrami operator of $M$ corresponding to eigenvalue $\lambda_{k}$.

Theorem B. [3]. Let $x: M \rightarrow E^{m}$ be an isometric immersion of a closed ndimensional Riemannan manifold $M$ into $E^{m}$. The total mean curvature of $x$ satısfies

$$
\int_{M} \alpha^{n} d v \geqq\left(\frac{\lambda_{1}}{n}\right)^{n / 2} \operatorname{vol}(M),
$$


SPECTRAL GEOMETRY OF CR-MINIMAL SUBMANIFOLDS

where $\operatorname{vol}(M)$ denotes the volume of $(M, g)$ and $\lambda_{1}$ denotes the first ergenvalue of the Laplace-Beltrami operator of $(M, g)$ acting on differentiable functions in $C^{\infty}(M)$. The equality holds if and only if there is a vector $c$ in $E^{m}$ such that $x-c$ is an imbedding of order 1.

COROLLARY 2.4. Let $M^{n}$ be a closed minmal submanfold of $C P^{m}$. Then we have

$$
\operatorname{vol}(M) \geqq c_{n} .
$$

Proof. Let $H$ be the mean curvature vector of $M^{n}$ in $H M(m+1)$. Let $H^{\perp}$ be the same as in lemma 2.1. Since $M^{n}$ is minimal in $\boldsymbol{C} P^{m}, H=H^{\perp}$. Now we use theorem A and lemma 2.1.

COROllary 2.5. Let $M^{2 n+p}$ be a closed CR-minumal submanifold of $C P^{m}$. Then we have

$$
\left[\frac{(2 n+p)^{2}+4 n+p}{2(2 n+p)^{2}}\right]^{n+p / 2} \operatorname{vol}(M) \geqq c_{2 n+p}
$$

The equality holds if and only if $M=C P^{1}$ is imbedded as a totally geodesic complex submanifold in $\boldsymbol{C P}^{m}$.

Proof. By using theorem A and lemma 2.1 we obtain (2.8).

We suppose that the equality holds. Then $M$ is isometric to a sphere of radius $R$. We have $\operatorname{vol}(M)=R^{2 n+p} c_{2 n+p}$, and then $R^{2}=2(2 n+p)^{2} /\left[(2 n+p)^{2}+4 n+p\right]$. Let $c$ and $r$ be the sectional curvature and the scalar curvature of $M$ respectively. Then $c=1 / R^{2}$ and

From corollary 2.3

$$
r=c(2 n+p-1)(2 n+p) .
$$

$$
r \leqq(1 / 4)\left[(2 n+p)^{2}+4 n-p\right] .
$$

From (2.9) and (2.10) we have

$$
\left[(2 n+p)^{2}+4 n\right](2 n+p-2)+p(6 n+3 p-2) \leqq 0 .
$$

But this occurs if and only if $n=1$ and $p=0$. Therefore $M$ is a unit 2-sphere imbedded as complex submanifold in $\boldsymbol{C} P^{m}$. Since $M$ and $C P^{m}$ have the same holomorphic sectional curvature $c=1$, we get that $M$ is totally geodesic in $C P^{m}$.

The converse is trivial because $\boldsymbol{C} P^{1}$ is imbedded in $H M(2)$ as a standard sphere.

The following corollaries can be obtained from theorem B and lemma 2.1.

COROLlary 2.6. Let $M^{n}$ be a closed minimal submanifold of $C P^{m}$. Then we have

$$
\lambda_{1} \leqq n
$$

COROllary 2.7. Let $M^{2 n+p}$ be a closed CR-minimal submanifold of $\boldsymbol{C P}^{m}$. 
Then we have

$$
\lambda_{1} \leqq\left[(2 n+p)^{2}+4 n+p\right] / 2(2 n+p) .
$$

In partıcular, if $M^{2 n}$ is a compact complex (resp. $M^{p}$ is a closed minmal totally real) submanifold of $\boldsymbol{C} P^{m}$ then

$$
\lambda_{1} \leqq n+1
$$

(resp. $\left.\lambda_{1} \leqq(p+1) / 2\right)$.

The following result gives a complete classification of the $C R$-minimal submanifolds of $C P^{m}$ which are minimal in some sphere of $H M(m+1)$.

THEOREM 2.8. Let $M^{2 n+p}$ be a CR-minimal submanifold of $C P^{m}$. Then $M^{2 n+p}$ is minimal in some sphere of $H M(m+1)$ if and only if one of the following cases holds:

a) $\quad p=0$ and $M^{2 n}$ is a totally geodesic complex submanifold of $\boldsymbol{C P}^{m}$.

b) $n=0$ and $M^{p}$ is a totally real submanifold of $C P^{m}$ for which there exists a totally geodesic complex submanifold $\bar{M}^{2 p}$ of $\mathrm{CP}^{m}$, such that $M^{p}$ is a totally real submanfold of $\bar{M}^{2 p}$.

Proof. We suppose that $M^{2 n+p}$ is minimal in a certain sphere $S$ of $H M(m+1)$. If $Q$ denotes the center of $S$, we can suppose that $Q$ is a diagonal matrix (otherwise we can use an isometry of $H M(m+1)$ of the type $A \mapsto P A P^{-1}$, where $P$ is in $U(m+1)$ ). Let $H$ be the mean curvature vector of $M$ in $H M(m+1)$. From the minimality of $M$ in $S$ we have $H=h \cdot(A-Q)$, for any $A$ in $M$ where $h$ is a real number with $h \neq 0$. It is clear that $Q \in T_{A}^{\perp}\left(\boldsymbol{C} P^{m}\right)$. Therefore $A Q=Q A$ for any $A$ in $M$. That is, $M$ is contained in the linear subspace, $L$ of $H M(m+1)$, which is defined by the equation $A Q=Q A$. We put

Then

$$
Q=\left(\begin{array}{ccccccc}
a_{1} & & & & & & \\
& \ddots & & & & & \\
& & a_{1} & & & \\
& & & \ddots & & & \\
& & & a_{r} & & \\
& & & & & \ddots & \\
& & & & & & a_{r}
\end{array}\right) .
$$

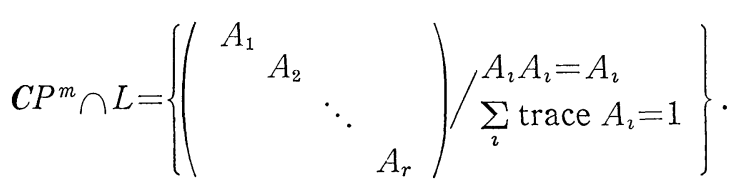

Since $A_{\imath} A_{\imath}=A_{\imath}$, we see that trace $A_{\imath}$ is a natural number. Hence for any $A$ in $C P^{m} \cap L$ there exists an index $j$ such that trace $A_{\jmath}=1$ and trace $A_{\imath}=0$ for all $\imath \neq \jmath$, which implies $A_{\imath}=0$ and 


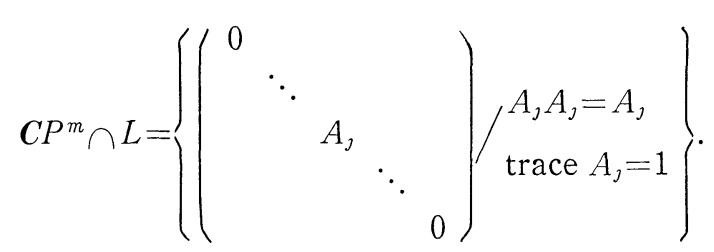

Therefore $M$ is contained in a connected component of $C P^{m} \cap L$. Each of these component is evidently a totally geodesic complex submanifold of $\boldsymbol{C} \boldsymbol{P}^{m}$ (it is a $\boldsymbol{C} P^{q}, q \leqq m$ ), and $M$ is a minimal submanifold of the sphere $S \cap L$. Consequently the problem is reduced to the study of $C R$-minimal submanifolds of $C P^{q}$ which are minimal in some sphere of $H M(q+1)$ whose center is $a I$ where $a$ is a real number and $I$ is the $(q+1) \times(q+1)$-identity matrix.

We have $H=h \cdot(A-a I)$. As $M$ is contained in the sphere we know that

$$
g(H, A-a I)=-1,
$$

and since $M$ is $C R$-minimal in $C P^{q}$,

$$
g(H, H)=\frac{(2 n+p)^{2}+4 n+p}{2(2 n+p)^{2}} .
$$

Therefore

$$
\begin{gathered}
h=-\frac{(2 n+p)^{2}+4 n+p}{2(2 n+p)^{2}}, \\
g(A-a I, A-a I)=\frac{2(2 n+p)^{2}}{(2 n+p)^{2}+4 n+p}
\end{gathered}
$$

for all $A$ in $M$. On the other hand,

$$
\begin{aligned}
g(A-a I, A-a I) & =g(A, A)-2 a g(A, I)+a^{2} g(I, I) \\
& =2(q+1) a^{2}-4 a+2 .
\end{aligned}
$$

From (2.16) and (2.17) we obtain

$$
(q+1)\left[(2 n+p)^{2}+4 n+p\right] a^{2}-2\left[(2 n+p)^{2}+4 n+p\right] a+4 n+p=0 .
$$

Since the discriminate of this equation must $\geqq 0$, we get

$$
(2 n+p)^{2}+4 n+p-(q+1)(4 n+p) \geqq 0,
$$

that is $(2 n+p)^{2} \geqq q(4 n+p)$. But $q \geqq n+p$, and so

$$
(2 n+p)^{2} \geqq(4 n+p)(n+p) .
$$

Therefore $4 n p \geqq 5 n p$, which implies $n=0$ or $p=0$.

*) Suppose $p=0$. Then we have $q=n$, that is $M^{2 n}$ is open in $C P^{n}$.

*) Suppose $n=0$. Then $p=q$. 
Conversely: If $M^{2 n}$ is a totally geodesic complex submanifold of $\boldsymbol{C} P^{m}$, then from proposition 1.6, $M$ is minimal in some sphere. Let $M^{p}$ be a totally real minimal submanifold of $C P^{p}$. For any $A \in M$, let $\left\{E_{1}, \cdots E_{p}\right\}$ be an orthonormal base of $T_{A}(M)$. Then we have that $\left\{E_{1}, \cdots E_{p}, J E_{1}, \cdots J E_{p}\right\}$ is an orthonormal base of $T_{A}\left(C P^{p}\right)$. Hence, if $H$ is the mean curvature vector of $M^{p}$ in $H M(p+1)$ it is easy to see from proposition 1.6, that

$$
H=\frac{1}{2 p}[I-(p+1) A]
$$

and so $M^{p}$ is minimal in some sphere.

(Q.E.D.)

COROLlary 2.9. Let $M^{2 n+p}$ be a closed $C R$-minimal submanifold of $\boldsymbol{C} P^{m}$. 1) If $M$ is in the cases a) or b) of theorem 2.8 , then $\left[(2 n+p)^{2}+4 n+p\right] / 2(2 n+p)$ is in $\operatorname{Spec}(M)$.

2) If $\lambda_{1}=\left[(2 n+p)^{2}+4 n+p\right] / 2(2 n+p)$, then $M$ is imbedded and is in the cases a) or b) of theorem 2.8, where $\operatorname{Spec}(M)$ is the spectrum of the Laplace-Beltrami operator of $M$ and $\lambda_{1}$ is the first eigenvalue of this operator.

Proof. 1) From the proof of theorem 2.8 and from a well know theorem of Takahashi [8], if $M$ is minimal in $S$ then $\lambda_{k}=\operatorname{dim}(M) / R^{2}$ for some $\lambda_{k}$ in $\operatorname{Spec}(M)$, where $R$ is the radius of $S$. Then $\lambda_{k}=\left[(2 n+p)^{2}+4 n+p\right] / 2(2 n+p)$.

2) From theorem $B$, we see, by choosing a suitable origen, that the immersion is an imbedding of order 1 . In particular it is minimal in some sphere, [8]. Now from theorem 2.8, $M$ is in the cases a) or b).

(Q.E.D.)

Corollary 2.10. Let $M^{2 n}$ be a complex compact submanifold of $\boldsymbol{C P}^{m}$. Then we have $\lambda_{1} \leqq n+1$. Moreover $M^{2 n}$ is totally geodesic in $\boldsymbol{C} P^{m}$ if and only if $\lambda_{1}=n+1$.

Proof. We consider corollaries 2.7 and 2.9 , and $\operatorname{Spec}\left(\boldsymbol{C} P^{n}\right)$, see [1].

COROLLARY 2.11. Let $M^{p}$ be a totally real closed minimal submanifold of $C P^{m}$. Then we have 1) If there exists $\bar{M}^{2 p}$ such that $\bar{M}^{2 p}$ is a totally geodesic complex submanifod of $\boldsymbol{C P}^{m}$ and $M^{p}$ is a totally real submanifold of $\bar{M}^{2 p}$, then $(p+1) / 2$ belongs to $\operatorname{Spec}\left(M^{p}\right)$.

2) If $\lambda_{1}=(p+1) / 2$, then there exists a totally geodesic complex submanifold $\bar{M}^{2 p}$ of $C P^{m}$ such that $M^{p}$ is a totally real submanifold of $\bar{M}^{2 p}$.

Proof. We consider corollary 2.9.

The author has known that corollaries 2.7 and 2.10 has been recently obtained by N. Ejiri. 


\section{REFERENCES}

[1] M. Berger, P. Gauduchon and E. Mazet, Le spectre d'une variété Riemanniene. Lecture Notes in Math. No. 194, Springer-Verlag, Berlin 1971.

[2] B. Y. Chen, Geometry of submanifolds. M. Dekker, New-York 1973.

[3] B.Y. CHEN, On the total curvature of immersed manifolds, IV: Spectrum and total mean curvature. Bull. Math. Acad. Sinıca, vol. 7 No. 3, 1979, 301-311.

[4] B. Y. CHEN, Geometry of submanıfolds and its applications. Science University of Tokyo, 1981.

[5] J.A. Little, Manifold with planar geodesic. J. Differential Geometry, 11, 1976, 265-285.

[6] S. Kobayashi and K. Nomizu, Foundations of Differential Geometry. WileyInterscience, New-York 1963.

[7] S.S. TAI, Minimum imbedding of compact symmetric spaces of rank one. J. Differential Geometry 2, 1968, 55-66.

[8] T. TAkaliashi, Minimal immersions of Riemannian manifolds. J. Math. Soc. Japan, 18, 1966, 380-385.

Departamento de Geometria y Topologia

FACultad de Ciencias

UNIVERSIDAD DE GRANADA

GRANADA (SPAIN) 